TRANSACTIONS OF THE

AMERICAN MATHEMATICAL SOCIETY

Volume 360, Number 3, March 2008, Pages $1377-1390$

S 0002-9947(07)04185-2

Article electronically published on October 3, 2007

\title{
TRUNCATIONS OF MULTILINEAR HANKEL OPERATORS
}

\author{
ALINE BONAMI, SANDRINE GRELLIER, AND MOHAMMAD KACIM
}

Abstract. We extend to multilinear Hankel operators the fact that some truncations of bounded Hankel operators are still bounded. We prove and use a continuity property of bilinear Hilbert transforms on products of Lipschitz spaces and Hardy spaces.

\section{Statement of the Results}

We prove that some truncations of bounded multilinear Hankel operators are bounded. This extends the same property for linear Hankel operators, a result obtained by $\mathrm{BB}$ (and independently in a particular case in $\mathrm{GB}$ ), which we first recall. A matrix $B=\left(b_{m n}\right)_{m, n \in \mathbb{N}}$ is called of Hankel type if $b_{m n}=b_{m+n}$ for some sequence $b \in l^{2}(\mathbb{N})$. We can identify $B$ with an operator acting on $l^{2}(\mathbb{N})$. Moreover, if we identify $l^{2}(\mathbb{N})$ with the complex Hardy space $\mathcal{H}^{2}(\mathbb{D})$ of the unit disc, then $B$ can be realized as the integral operator, called a Hankel operator with symbol $b$ and denoted by $H_{b}$, which acts on $f \in \mathcal{H}^{2}(\mathbb{D})$ by

$$
H_{b} f(z)=\frac{1}{2 \pi} \int_{\partial \mathbb{D}} \frac{b(\zeta) f(\bar{\zeta})}{1-\bar{\zeta} z} d \sigma(\zeta), z \in \mathbb{D} .
$$

Here $d \sigma$ is the Euclidean measure on the unit circle. The symbol $b$ is given by $b(\zeta):=\sum_{k=0}^{\infty} b_{k} \zeta^{k}$. In other words, $H_{b} f=\mathcal{C}(b \check{f})$ where $\mathcal{C}$ denotes the Cauchy integral, and $\check{f}(\zeta):=f(\bar{\zeta})$. If $f(\zeta)=\sum_{n \in \mathbb{N}} a_{n} \zeta^{n}$, one has

$$
H_{b} f(z)=\sum_{m \in \mathbb{N}}\left(\sum_{n \in \mathbb{N}} a_{n} b_{m+n}\right) z^{m} .
$$

Now, we consider truncations of matrices, which are defined as follows. For $\beta, \gamma \in \mathbb{R}$, the truncated matrix $\Pi_{\beta, \gamma}(B)$ is the matrix whose $(m, n)$ entry is $b_{m n}$ or zero, depending on the fact that $m \geq \beta n+\gamma$ or not. It is proved in $\mathrm{BB}$. that such truncations, for $\beta \neq-1$, preserve the boundedness for Hankel operators. The proof consists in showing that truncations are closely related to bilinear periodic Hilbert transforms. One then uses the theorem of Lacey-Thiele (see [LT1, [LT2], [LT3]) in the periodic setting.

Received by the editors November 22, 2004 and, in revised form, October 7, 2005.

2000 Mathematics Subject Classification. Primary 47B35; Secondary 42A50, 47A63, 47B10, 47B49.

Key words and phrases. Hankel operator, truncation, Hardy spaces, Lipschitz spaces, bilinear Hilbert transform.

The authors were partially supported by the 2002-2006 IHP Network, Contract Number: HPRN-CT-2002-00273 - HARP.

The authors would like to thank Joaquim Bruna who suggested this problem. 
We are interested in the same problem, but for multilinear Hankel operators. For $n \in \mathbb{N}$, we define the multilinear Hankel operator $H_{b}^{(n)}$ as follows. For $f_{1}, \ldots, f_{n} \in$ $\mathcal{H}^{2}(\mathbb{D})$, let

$$
\begin{aligned}
H_{b}^{(n)}\left(f_{1}, \ldots, f_{n}\right)(z) & =\frac{1}{2 \pi} \int_{\partial \mathbb{D}} \frac{b(\zeta) f_{1}(\bar{\zeta}) \ldots f_{n}(\bar{\zeta})}{1-\bar{\zeta} z} d \sigma(\zeta) \\
& =H_{b}\left(f_{1} \times \cdots \times f_{n}\right)(z) \\
& =\sum_{i_{0}, \ldots, i_{n}} b_{m+i_{1}+\cdots+i_{n}} a_{i_{1}}^{1} \ldots a_{i_{n}}^{n} z^{m}
\end{aligned}
$$

whenever $f_{j}(z)=\sum a_{i}^{j} z^{i}$. This last expression means that, when equipped with the canonical basis of $\mathcal{H}^{2}(\mathbb{D})$, the matrix of the operator, whose entries are indexed in $\mathbb{N}^{n+1}$, are given by $b_{i_{0}+\cdots+i_{n}}, i_{0}, \ldots, i_{n} \in \mathbb{N}$. The truncated operator is obtained when truncating this matrix as follows. For $\beta=\left(\beta_{1}, \ldots, \beta_{n}\right) \in \mathbb{R}^{n}$ and $\gamma \in \mathbb{R}$, we denote by $\Pi_{\beta, \gamma}\left(H_{b}^{(n)}\right)$ the operator with $(n+1)$-dimensional matrix whose $\left(i_{0}, \ldots, i_{n}\right)$-entry is $b_{i_{0}+\cdots+i_{n}}$ if $\beta_{1} i_{1}+\cdots+\beta_{n} i_{n}+\gamma \leq i_{0}$ and zero otherwise. Our main result is the following.

Theorem 1. If $H_{b}^{(n)}$ is a continuous multilinear Hankel operator from $\left(\mathcal{H}^{2}(\mathbb{D})\right)^{n}$ into $\mathcal{H}^{2}(\mathbb{D})$, then so are its truncated operators $\Pi_{\beta, \gamma}\left(H_{b}^{(n)}\right)$ for any $\beta=\nu(1, \ldots, 1)$, $\gamma \in \mathbb{R}$. Furthermore, there exists a constant $C=C(\nu)$ uniformly bounded when $\nu$ lies in a compact set of $\mathbb{R} \backslash\{-1,0\}$ so that

$$
\left\|\Pi_{\beta, \gamma}\left(H_{b}^{(n)}\right)\right\|_{\left(\mathcal{H}^{2}(\mathbb{D})\right)^{n} \rightarrow \mathcal{H}^{2}(\mathbb{D})} \leq C\left\|H_{b}^{(n)}\right\|_{\left(\mathcal{H}^{2}(\mathbb{D})\right)^{n} \rightarrow \mathcal{H}^{2}(\mathbb{D})} .
$$

Theorem 1 is deduced from an estimate on the bilinear Hilbert transform in the periodic setting, which is of independent interest. Let us first give some notations. The usual Lipschitz spaces of order $\alpha$ of $2 \pi$-periodic functions are denoted by $\Lambda_{\alpha}(\mathbb{T})$, while $H^{p}(\mathbb{T})$ denotes the real Hardy space, $p>0$. Here $\mathbb{T}:=\mathbb{R} / 2 \pi \mathbb{Z}$ denotes the torus.

Let $b \in \Lambda_{\alpha}(\mathbb{D})$ and $f \in \mathcal{H}^{q}(\mathbb{D})$. The boundary values of such functions may be seen either as functions on the set of complex numbers of modulus 1 , or on the torus $\mathbb{T}=\mathbb{R} / 2 \pi \mathbb{Z}$ (equivalently as $2 \pi$-periodic functions on $\mathbb{R}$ ). We write $f\left(e^{i t}\right)$ or $f(t)$ depending on the context, and hope that it does not introduce any confusion.

Let us recall that, for $f$ and $b$ trigonometric polynomials on the torus, the periodic bilinear Hilbert transform of $f$ and $b$ is given, for $k, l \in \mathbb{Z}, k \neq-l, l \neq 0$, by

$$
\mathcal{H}_{k, l}(b, f)(x)=p . v \cdot \int_{\mathbb{T}} b(k x+l t) f(t) \frac{d t}{\tan \frac{x-t}{2}} .
$$

Lacey-Thiele's Theorem, once transferred to the periodic setting, is the following.

Theorem 2 ([BB] $)$. Let $1<p, q \leq \infty$ with $\frac{1}{r}=\frac{1}{p}+\frac{1}{q}<\frac{3}{2}$. Then, for any $k, l \in \mathbb{Z}, k \neq-l$ and $l \neq 0$, there exists a constant $C=C(k, l)>0$ so that, for any $f \in L^{p}(\mathbb{T})$ and any $b \in L^{q}(\mathbb{T})$,

$$
\left\|\mathcal{H}_{k, l}(b, f)\right\|_{L^{r}(\mathbb{T})} \leq C(k, l)\|f\|_{L^{p}(\mathbb{T})}\|b\|_{L^{q}(\mathbb{T})} .
$$

Furthermore, $C(k, l)$ is uniformly bounded when $k / l$ lies in a compact set of $\mathbb{R} \backslash$ $\{-1,0\}$. 
Remark. The recent work of Grafakos and Li (see GraL) allows us to have uniform constants outside a neighborhood of -1 "in most cases", in particular when $r=$ $p=2$.

We adapt the definition to our setting, and define, for $k, l, \mu \in \mathbb{Z}$ with $k \neq-l$, $l \neq 0$ and $\mu \in[-l, l]$,

$$
\mathcal{H}_{k, l, \mu}(b, f)(x)=\int_{\mathbb{T}}\left(b(k x+l t) e^{i \mu(x-t)}-b((k+l) x)\right) f((k+l) t) \frac{d t}{\tan \frac{x-t}{2}} .
$$

We prove the following.

Theorem 3. Let $1<p<\infty, 0<q<p$ and $\alpha=\frac{1}{q}-\frac{1}{p}$. Let $k, l, \mu \in \mathbb{Z}$, with $k \neq-l, l \neq 0$ and $\mu \in[-l, l]$. There exists a constant $C=C(k, l)>0$ so that, for any sufficiently smooth functions $b \in \Lambda_{\alpha}(\mathbb{T})$ and $f \in H^{q}(\mathbb{T})$

$$
\left\|\mathcal{H}_{k, l, \mu}(b, f)\right\|_{H^{p}(\mathbb{T})} \leq C\|f\|_{H^{q}(\mathbb{T})}\|b\|_{\Lambda_{\alpha}(\mathbb{T})} .
$$

Furthermore, $C(k, l)$ is independent of $\mu$ and uniformly bounded when $k / l$ lies in a compact set of $\mathbb{R} \backslash\{-1,0\}$.

In the following, we say that a constant $C(k, l)$ is admissible when it is uniformly bounded when $k / l$ lies in a compact set of $\mathbb{R} \backslash\{-1,0\}$.

The difficulty, here, is the uniform bound of constants. We remark that the limiting case $b \in L^{\infty}(\mathbb{T})$ is given by the Lacey-Thiele Theorem, that is, Theorem 2. We will also give a non-periodic version of Theorem 3. Our methods rely on the ordinary Calderòn-Zygmund theory in a bilinear setting for the local part (one may consult [GraK] and [GraT]), and on transference for the non-local part.

We do not know whether the constants $C(k, l)$ may be chosen uniformly bounded outside a neighborhood of $\frac{k}{l}=-1$ as in Theorem 2. We only prove that it is the case for the non-periodic version of Theorem 3 (see Proposition [13).

Let us come back to holomorphic functions and to truncations. Denote by $\Lambda_{\alpha}(\mathbb{D})$, $\alpha>0$, the space of functions that are holomorphic in $\mathbb{D}$ and whose boundary values are in $\Lambda_{\alpha}(\mathbb{T})$. Also denote by $\mathcal{H}^{p}(\mathbb{D})$ the complex Hardy space on the unit disc, $p>0$. Recall that, for $p<1$, the dual of $\mathcal{H}^{p}(\mathbb{D})$ is $\Lambda_{\alpha}(\mathbb{D})$, with $p=(\alpha+1)^{-1}([\mathbb{D}])$. As an easy consequence of duality and factorization, one obtains that the Hankel operator $H_{b}$ is bounded from $\mathcal{H}^{q}(\mathbb{D})$ into $\mathcal{H}^{p}(\mathbb{D})$, with $q<p$ and $p>1$, if and only if the symbol $b$ is in $\Lambda_{\alpha}(\mathbb{D})$ with $\alpha=\frac{1}{q}-\frac{1}{p}$. Moreover, there exists a constant $C$ such that, for all holomorphic polynomials $f$,

$$
\left\|H_{b}(f)\right\|_{\mathcal{H}^{p}(\mathbb{D})} \leq C\|b\|_{\Lambda_{\alpha}(\mathbb{D})} \times\|f\|_{\mathcal{H}^{q}(\mathbb{D})} .
$$

Theorem 3 has the following corollary, which gives the link with truncations.

Corollary 4. Let $1<p<\infty$ and $0<q<p$. Let $\alpha=\frac{1}{q}-\frac{1}{p}$ and $b \in \Lambda_{\alpha}(\mathbb{D})$. Then, for any $\beta, \gamma \in \mathbb{R}$ with $\beta \neq-1$, the operator $\Pi_{\beta, \gamma}\left(H_{b}\right)$ is continuous from $\mathcal{H}^{q}(\mathbb{D})$ into $\mathcal{H}^{p}(\mathbb{D})$. More precisely, there exists a constant $C$ uniformly bounded for $\gamma \in \mathbb{R}$ and $\beta$ in a compact set of $\mathbb{R} \backslash\{-1,0\}$ so that

$$
\left\|\Pi_{\beta, \gamma}\left(H_{b}\right)\right\|_{\mathcal{H}^{q}(\mathbb{D}) \rightarrow \mathcal{H}^{p}(\mathbb{D})} \leq C\left\|H_{b}\right\|_{\mathcal{H}^{q}(\mathbb{D}) \rightarrow \mathcal{H}^{p}(\mathbb{D})} .
$$

So, if $H_{b}$ is a bounded operator from $\mathcal{H}^{q}(\mathbb{D})$ into $\mathcal{H}^{p}(\mathbb{D})$, its truncates $\Pi_{\beta, \gamma}\left(H_{b}\right)$ are also bounded. 
Remark 5. For $\beta=-1$, we are interested in the behavior of the norm of the operator $\Pi_{-1, N}\left(H_{b}\right)$, for $N$ a positive integer. Then $\left(I-\Pi_{-1, N}\right)\left(H_{b}\right)=H_{S_{N-1}(b)}$ where $S_{N}$ gives the $N$-th partial sum of the Fourier series, that is, the convolution operator corresponding to the Dirichlet kernel. Then, for $1<p<\infty, 0<q<p$, $\alpha=\frac{1}{q}-\frac{1}{p}$ and $b \in \Lambda_{\alpha}(\mathbb{D})$, one has

$$
\left\|\Pi_{-1, \gamma}\left(H_{b}\right)\right\|_{\mathcal{H}^{q}(\mathbb{D}) \rightarrow \mathcal{H}^{p}(\mathbb{D})} \leq C \log N \times\left\|H_{b}\right\|_{\mathcal{H}^{q}(\mathbb{D}) \rightarrow \mathcal{H}^{p}(\mathbb{D})} .
$$

Moreover, the best constant in the previous inequality is bounded below by $c \log N$, with $c$ independent of $N$.

This result follows from the fact that $\left\|S_{N}\right\|_{\Lambda_{\alpha} \mapsto \Lambda_{\alpha}} \simeq \log N$. We will prove it in the next section for the convenience of the reader.

We use the notation $A \simeq B$ whenever there exist two universal constants $c, c^{\prime}>0$ so that $c^{\prime} B \leq A \leq c B$.

Remark 6 . For $\beta=0$, it is easy to show that truncations uniformly preserve the class of bounded Hankel operators. It is sufficient to consider positive integer values of $\gamma$. In fact, one has $\Pi_{0, N+1}\left(H_{b} f\right)=\left(I-S_{N}\right) H_{b} f$ for $N \in \mathbb{N}$ so that it suffices to use the fact that $S_{N}$ is a bounded operator on $\mathcal{H}^{p}(\mathbb{D}), 1<p<\infty$, with a bound independent on $N$. When $\beta=\infty$ (that is, when truncations of Hankel matrices are with respect to directions $n=N)$, one may also show the analogous fact. To do so, it suffices to argue by duality and to show that, for $b \in \Lambda_{\alpha}(\mathbb{D}), \Pi_{0, N}\left(H_{b}\right)$ maps boundedly $\mathcal{H}^{p^{\prime}}(\mathbb{D})$ into $\Lambda_{\alpha^{\prime}}(\mathbb{D})$, where $\alpha^{\prime}=\alpha-1 / p^{\prime}$, with a bound independent of $N$. We will prove this in the next section.

Let us deduce Theorem 1 from the corollary. It is clear that

$$
H_{b}^{(n)}\left(f_{1}, \ldots, f_{n}\right)(z)=H_{b}\left(f_{1} \times \cdots \times f_{n}\right)(z) .
$$

Using the factorization of functions in Hardy classes, we know that $H_{b}^{(n)}$ is bounded as an operator from $\left(\mathcal{H}^{2}(\mathbb{D})\right)^{n}$ into $\mathcal{H}^{2}(\mathbb{D})$ if and only if the Hankel operator $H_{b}$ is bounded from $\mathcal{H}^{2 / n}(\mathbb{D})$ into $\mathcal{H}^{2}(\mathbb{D})$, that is, if and only if $b$ is in $\Lambda_{\alpha}(\mathbb{D})$ for $\alpha=\frac{n-1}{2}$. To conclude, we use the fact that the truncation $\Pi_{\beta, \gamma}$ of $H_{b}^{(n)}$ for $\beta=\nu(1, \ldots, 1)$, corresponds to the truncation $\Pi_{\nu, \gamma}$ of $H_{b}$, as it can be easily verified.

Let us finally remark that this paper leaves open the general problem of all truncations of multilinear Hankel operators. One may be tempted to reduce to multilinear Hilbert transforms, as studied in [MTT].

The remainder of the paper is organized as follows. In the next section, we deduce the corollary from Theorem 3, In the last one, we prove Theorem 1, Let us emphasize the fact that this last proof does not use Lacey-Thiele Theorem, and is elementary compared to it.

\section{The Link Between truncations and Bilinear Hilbert transforms}

At first, we prove the result corresponding to truncations with $\beta=\infty$, as stated in Remark [6.

Proof. Let us recall that it is sufficient to prove that, for $\alpha^{\prime}=\alpha-\frac{1}{p^{\prime}}$,

$$
\left\|\Pi_{0, N} H_{b}(f)\right\|_{\Lambda_{\alpha^{\prime}}(\mathbb{D})} \leq C\|f\|_{\mathcal{H}^{p^{\prime}}(\mathbb{D})}\|b\|_{\Lambda_{\alpha}(\mathbb{D})}
$$


with a constant independent of $N \in \mathbb{N}$. By density, it is sufficient to prove this inequality on trigonometric polynomials. It is easy to show that

$$
T f(z):=\Pi_{0, N} H_{b}(f)(z)=\frac{1}{2 \pi} \int_{\partial \mathbb{D}} b(\zeta) \check{f}(\zeta)(z \bar{\zeta})^{N} \frac{d \sigma(\zeta)}{1-z \bar{\zeta}},
$$

where $\check{f}(\zeta):=f(\bar{\zeta})$ as before. Using Stokes Formula, we get

$$
T f(z)=\frac{1}{\pi} \int_{\mathbb{D}}\left(b\left(r e^{i \theta}\right)+b^{\prime}\left(r e^{i \theta}\right)\right) f\left(r e^{-i \theta}\right) z^{N} r^{N} e^{-i N \theta} \frac{d \theta d r}{1-z r e^{-i \theta}} .
$$

Assume to simplify that $0<\alpha<1$. By the holomorphic characterization of Lipschitz spaces, it suffices to show that

$$
\left|(T f)^{\prime}(z)\right| \leq C(1-|z|)^{\alpha^{\prime}-1}
$$

Computing the derivative of $T(f)$, and using the fact that $\left|N z^{N} r^{N}\right| \leq C(1-r|z|)^{-1}$, we get the estimate

$$
\left|(T f)^{\prime}(z)\right| \leq C \int_{\mathbb{D}}\left|b\left(r e^{i \theta}\right)+b^{\prime}\left(r e^{i \theta}\right)\right|\left|f\left(r e^{-i \theta}\right)\right|(1-r|z|)^{-1} \frac{d \theta d r}{\left|1-z r e^{-i \theta}\right|} .
$$

We use that $\left|b\left(r e^{i \theta}\right)+b^{\prime}\left(r e^{i \theta}\right)\right| \leq C\|b\|_{\Lambda_{\alpha}(\mathbb{D})}(1-r)^{\alpha-1}$ and Hölder's Inequality to get that

$$
\begin{aligned}
\left|(T f)^{\prime}(z)\right| & \leq C\|b\|_{\Lambda_{\alpha}(\mathbb{D})}\|f\|_{L^{p^{\prime}}(\mathbb{T})} \int_{0}^{1} \frac{(1-r)^{\alpha-1}}{(1-r|z|)^{2-1 / p}} d r \\
& \leq C(1-|z|)^{\alpha-1+1 / p}\|b\|_{\Lambda_{\alpha}(\mathbb{D})}\|f\|_{\mathcal{H}^{p^{\prime}(\mathbb{D})}} .
\end{aligned}
$$

It ends the proof.

We now prove that $\left\|S_{N}(f)\right\|_{\Lambda_{\alpha} \rightarrow \Lambda_{\alpha}} \simeq \log N$. We first recall the following characterization of Lipschitz spaces.

Proposition 7. A function b belongs to $\Lambda_{\alpha}(\mathbb{T})$ if and only if there exists a decomposition $b=\sum_{j=0}^{\infty} b_{j}$ with $\left\|\nabla^{l} b_{j}\right\|_{\infty} \leq A 2^{-j(\alpha-l)}$ for any integer $0 \leq l \leq k$, where $k$ is the smallest integer $>\alpha$.

If $b \in \Lambda_{\alpha}(\mathbb{D})$, the decomposition may be chosen so that the spectrum of $b_{j}, j \geq 1$, lies in $\left\{2^{j-1}, \ldots, 2^{j+2}\right\}$ and $b_{0}$ is a constant. Moreover, the norm is equivalent to the smallest constant $A$ for which we have $\left\|b_{j}\right\|_{\infty} \leq A 2^{-j \alpha}$ for all $j \geq 0$.

The first statement can be found in $[\mathbf{S}$, on page 256. The second one can be obtained from the proof given there when $\alpha<1$, using a variant of the kernel of De La Vallée Poussin adapted to the dyadic decomposition. Then one concludes using the action of powers of the Laplacian, which give isomorphisms between Lipschitz spaces with different parameters.

As a classical corollary, we prove the following.

Corollary 8. For any $N \in \mathbb{N}$, the norm of the operator $S_{N}$ from $\Lambda_{\alpha}(\mathbb{T})$ into itself is equivalent to $\log N$. In other words

$$
\left\|S_{N}\right\|_{\Lambda_{\alpha}(\mathbb{T}) \mapsto \Lambda_{\alpha}(\mathbb{T})} \simeq \log N .
$$

Proof. Denote by $D_{N}$ the Dirichlet kernel of order $N$. As $\left\|D_{N}\right\|_{L^{1}(\mathbb{T})} \simeq \log N$, it follows easily that

$$
\left\|S_{N}\right\|_{\Lambda_{\alpha}(\mathbb{T}) \mapsto \Lambda_{\alpha}(\mathbb{T})} \leq C \log N .
$$


It is sufficient to prove the converse inequality for the operator $S_{N}^{+}$defined by

$$
S_{N}^{+} f(t)=\sum_{n \leq N} \widehat{f}(n) e^{i n t} .
$$

We start from the well known fact that, for all $M \geq 1$, one can find a trigonometric polynomial $g$ of degree $2 M$ such that $\|g\|_{L^{\infty}(\mathbb{T})} \simeq 1$ and $\left\|S_{M}^{+} g\right\|_{L^{\infty}(\mathbb{T})} \simeq \log M$. Let us assume that $N=4 \times 2^{n}$ for some integer $n \geq 1$. Take $M=\frac{N}{4}$; then the function $f=e^{i 3 \frac{N}{4} t} g$ satisfies $\|f\|_{L^{\infty}(\mathbb{T})} \simeq 1$ and $\left\|S_{N}^{+} f\right\|_{L^{\infty}(\mathbb{T})} \simeq \log N$ and has a spectrum included in $\left\{2^{n}, \ldots, 2^{n+3}\right\}$. Moreover, by the previous proposition, and the spectrum assumption on $f$, one has $\|f\|_{\Lambda_{\alpha}(\mathbb{T})} \simeq 2^{n \alpha}\|f\|_{L^{\infty}(\mathbb{T})}$ and $\left\|S_{N}^{+} f\right\|_{\Lambda_{\alpha}(\mathbb{T})} \simeq$ $2^{n \alpha}\left\|S_{N}^{+} f\right\|_{L^{\infty}(\mathbb{T})}$. This finishes the proof.

In the following, we are going to use the same kind of idea in the general case to get rid of the dependence in $\gamma$ of the constants. Let us now consider the truncation $\Pi_{\beta, \gamma}$. Remark that, if $\beta$ and $\gamma$ are negative, then $\Pi_{\beta, \gamma} H_{b}=H_{b}$ and there is nothing to prove. We will not consider this case any longer. If $\gamma<0$ and $\beta>0$, then $\left(I-\Pi_{\beta, \gamma}\right) H_{b}=\left(I-\Pi_{\beta, \gamma}\right) H_{P_{[-\gamma / \beta]}(b)}$ where $P_{N}(b)$ denotes the projection of $b$ on the space of functions whose spectrum is contained in $[N, \infty)$. If $\gamma>0$ and $\beta>0$, then $\Pi_{\beta, \gamma} H_{b}=\Pi_{\beta, \gamma} H_{P_{[\gamma]}(b)}$. In the last case, where $\gamma>0$ and $\beta<0$, then $\Pi_{\beta, \gamma} H_{b}=\Pi_{\beta, \gamma} H_{P_{n \beta, \gamma}(b)}$ where $n^{\beta, \gamma}=\min \{[\gamma],[-\gamma / \beta]\}$ (here [.] denotes the integer part). So, in any case, $b$ may be replaced by some projection $P_{n^{\beta, \gamma}}(b)$ of $b$ where

$$
n^{\beta, \gamma}=\left\{\begin{array}{l}
{[-\gamma / \beta] \text { when } \gamma<0 \text { and } \beta>0,} \\
{[\gamma] \text { when } \gamma>0 \text { and } \beta>0,} \\
\min \{[\gamma],[-\gamma / \beta]\}\} \text { otherwise. }
\end{array}\right.
$$

So, it suffices to consider the symbols $b$ modulo a polynomial of degree smaller than $n^{\beta, \gamma}$. The following lemma will allow us to modify $b$ by adding such a polynomial. The main point, here, is the fact that the constant is admissible.

Lemma 9. Let $\alpha>0$. There exists a constant $C$ with the following property: for any $b \in \Lambda_{\alpha}(\mathbb{D})$ and $N \in \mathbb{N}$, there exists a function $\tilde{b}$ such that $P_{N}(\tilde{b})=P_{N}(b)$, and, for all $M \in \mathbb{Z}$,

$$
\left\|\tilde{b} \zeta^{M}\right\|_{\Lambda_{\alpha}(\mathbb{T})} \leq C\left(\frac{|M|}{N+1}+1\right)^{\alpha}\|b\|_{\Lambda_{\alpha}(\mathbb{D})} .
$$

Proof. When $N \leq 16$, we take $\tilde{b}=b$. We conclude easily, using the fact that the norm in $\Lambda_{\alpha}(\mathbb{T})$ of $\zeta \rightarrow \zeta^{M}$ is equivalent to $(|M|+1)^{\alpha}$. Now consider $N>16$, and choose $N_{0}$ so that $2^{N_{0}} \leq N<2^{N_{0}+1}$. Use the Littlewood-Paley decomposition given in Proposition 7 to write $b$ as $b=\sum b_{j}$ with the spectrum of $b_{j}$, for $j \geq 1$, included in $\left[2^{j-1}, 2^{j+2}\right)$. We can clearly take

$$
\tilde{b}=\sum_{j \geq N_{0}-2} b_{j} .
$$

For simplicity, we assume that $\alpha<1$. The new function $a(\zeta)=\tilde{b}(\zeta) \zeta^{M}$ can be written as $\sum_{j \geq N_{0}-2} a_{j}$, with $a_{j}(\zeta)=b_{j}(\zeta) \zeta^{M}$. It is clear that, for $j \geq N_{0}-2$,

$$
\left\|a_{j}\right\|_{\infty} \leq C 2^{-j \alpha}, \quad\left\|a_{j}^{\prime}\right\|_{\infty} \leq C\left(\frac{|M|}{N+1}+1\right) 2^{j(1-\alpha)} .
$$


Indeed, for the second inequality, we use the Bernstein Inequality to get that $\left\|b_{j}^{\prime}\right\|_{\infty} \leq C 2^{j}\left\|b_{j}\right\|_{\infty}$. The second term, in the derivative of $a_{j}$, is bounded by $|M| \times\left\|b_{j}\right\|_{\infty}$. The inequality follows at once, using the fact that $N+1 \leq 2^{j+3}$. We adapt the proof given in $[\mathrm{S}]$ to deduce the required inequality for $a$.

Proof. We are now in a position to prove that Corollary 4 follows from Theorem 3 , It is sufficient to prove, for trigonometrical polynomials $b$ and $f$, that

$$
\left\|\Pi_{\beta, \gamma} H_{b}(f)\right\|_{\mathcal{H}^{p}(\mathbb{D})} \leq C\|b\|_{\Lambda_{\alpha}(\mathbb{D})} \times\|f\|_{\mathcal{H}^{q}(\mathbb{D})}
$$

for some constant $C$ that depends only on $\beta$ and is uniformly bounded when $\beta$ lies in a compact set of $\mathbb{R} \backslash\{-1,0\}$. Moreover, since $b$ and $f$ are trigonometric polynomials, we can restrict ourselves to rational values $\frac{k}{l}$ for $\beta$, and values of $\gamma$ such that $\gamma l$ is an integer, as soon as we show that the constant $C$ may be taken independent of $k, l, \gamma$ when these last ones vary in such a way that $\frac{k}{l}$ lies in a compact set of $\mathbb{R} \backslash\{-1,0\}$. So, we assume that $\beta=\frac{k}{l} \neq-1$ with $k, l \in \mathbb{Z}, l \neq 0$ and that $l \gamma \in \mathbb{Z}$. We may also assume that $k+l>0$.

Assume that $f(t)=\sum_{n \in \mathbb{N}} a_{n} e^{i n t}$. An elementary computation shows that the analytic part of the function

$$
p . v . \int_{\mathbb{T}} b(k x+l t) e^{i \gamma l(x-t)} \check{f}((k+l) t) \frac{d t}{\tan \frac{x-t}{2}}
$$

is equal to

$$
\sum_{n, m \in \mathbb{N}} a_{n} b_{m+n} \operatorname{sign}(l(m-\beta n-\gamma)) e^{i m(k+l) x} .
$$

We recognize the function $\operatorname{sign}(l)\left(2 \Pi_{\beta, \gamma}-I\right)\left(H_{b}(f)\right)\left(e^{i(k+l) \cdot}\right)$, for which we want to have an $L^{p}$ estimate (recall that we have such an estimate for $H_{b}(f)$ ). By Lemma 9 and the remark before, we can replace $b$ by the function $\tilde{b}$ that corresponds to the choice $N=n^{\beta, \gamma}$. On the other hand, this term is equal to the analytic part of the function

$$
e^{i[\gamma](l+k) x} \int_{\mathbb{T}} \underbrace{\tilde{b}(k x+l t) e^{-i[\gamma](k x+l t)}}_{=\hat{b}(k x+l t)} e^{i \delta l(x-t)} \check{f}((k+l) t) \frac{d t}{\tan \frac{x-t}{2}}
$$

where $\delta=\gamma-[\gamma]$. This is the analytic part of $e^{i[\gamma](l+k) x} \mathcal{H}_{k, l, \delta l}(\hat{b}, f)$, up to the Cauchy projection of

$$
\tilde{b}((k+l) x) \times p \cdot v \cdot \int_{\mathbb{T}} \check{f}((k+l) t) \frac{d t}{\tan \frac{x-t}{2}} .
$$

This last term coincides with $H_{\tilde{b}}(f)\left(e^{i(k+l) x}\right)$ and is in $\mathcal{H}^{p}(\mathbb{D})$ with norm bounded by

$$
C\|\tilde{b}\|_{\Lambda_{\alpha}(\mathbb{D})} \times\|f\|_{\mathcal{H}^{q}(\mathbb{D})} \leq C\|b\|_{\Lambda_{\alpha}(\mathbb{D})} \times\|f\|_{\mathcal{H}^{q}(\mathbb{D})} .
$$

By Theorem [3, the term $e^{i[\gamma](l+k) x} \mathcal{H}_{k, l, \delta}(\hat{b}, f)$ is also in $H^{p}(\mathbb{T})$ with norm bounded by $C\|\hat{b}\|_{\Lambda_{\alpha}(\mathbb{T})}\|f\|_{H^{q}(\mathbb{T})}$ for some admissible constant $C$. This last quantity is, in turn, bounded by $C(|\beta|+1)^{\alpha}\|b\|_{\Lambda_{\alpha}(\mathbb{D})} \times\|f\|_{\mathcal{H}^{q}(\mathbb{D})}$, as one can see by using Lemma 9. 


\section{Proof of Theorem 3}

Let us note $L=k+l$. Let us first remark that it is sufficient to consider the case when $L>0$ (the general case follows by replacing $b$ by $\tilde{b}$ such that $\tilde{b}(x):=b(-x)$ and $f$ by $\tilde{f}$ such that $\tilde{f}(x):=f(-x))$.

Let us give a first reduction of the problem. We use the following lemma, where $\tau$ denotes the translation by $y$ on the torus. Its proof is elementary.

Lemma 10. For all trigonometrical polynomials $b$ and $f$, the following identity is valid:

$$
\mathcal{H}_{k, l, \mu}\left(b \circ \tau^{L}, f \circ \tau\right)=\mathcal{H}_{k, l, \mu}(b, f) \circ \tau .
$$

Cutting into eight parts the function $f$ and using this lemma, we reduce to periodic functions with support in $\left(0,+\frac{\pi}{4}\right)$ in the case $q>1$. For the case of $q \leq 1$, by the atomic decomposition theorem of $H^{q}(\mathbb{T})$, it suffices to consider the action of $\mathcal{H}_{k, l, \mu}(b, \cdot)$ on $H^{q}(\mathbb{T})$-atoms, also called $q$-atoms. So, let $f$ be a $q$-atom, that is, either a constant or a function $f$ on $\mathbb{T}$ supported in some interval $I$ on the torus (we will always assume its length is less than $\pi / 4$, which is possible) so that

$$
\|f\|_{L^{\infty}(\mathbb{T})} \leq|I|^{-1 / q} \text { and } \int_{\mathbb{T}} x^{k} f(x) d x=0 \text { for any integer } k \leq \frac{1}{q}-1 .
$$

We have seen that $\mathcal{H}_{k, l, \mu}(b, f)$ is $2 \pi / L$-periodic. We simplify the notation, and write

$$
T f(x):=\mathcal{H}_{k, l, \mu}(b, f)\left(\frac{x}{L}\right)
$$

so that, after a change of variable in the integral, one can write

$$
T f(x)=\int_{-\pi L}^{\pi L}\left[b\left(x+\frac{l}{L}(t-x)\right) e^{i \frac{\mu}{L}(x-t)}-b(x)\right] f(t) \frac{d t}{L \tan \frac{x-t}{2 L}} .
$$

We are restricted to prove that

$$
\|T(f)\|_{L^{p}(]-\pi, \pi[)} \leq C\|b\|_{\Lambda_{\alpha}(\mathbb{T})}\|f\|_{H^{q}(\mathbb{T})}
$$

for $f$ supported in the interval $\left(0,+\frac{\pi}{4}\right)$ when $q>1$, or

$$
\|T(f)\|_{L^{p}(]-\pi, \pi[)} \leq C\|b\|_{\Lambda_{\alpha}(\mathbb{T})}\|f\|_{H^{q}(\mathbb{T})}
$$

for $f$ a $q$-atom supported in $(0,+r)$ for some $r \leq \frac{\pi}{4}$ when $q \leq 1$.

Let us explain the next reduction of the problem. For $|x|<\pi,|s|<L \pi$ and $s$ in the support of the periodic function $f$, we can write

$$
\frac{1}{L \tan \frac{x-s}{2 L}}=\frac{1}{x-s}+\frac{1}{L} \psi\left(\frac{x-s}{L}\right)
$$

with $\psi$ a $\mathcal{C}^{\infty}$ function with compact support. Indeed, the conditions on $s, x$ and $L$, imply that $\frac{|x-s|}{L} \leq \frac{3 \pi}{2}$. We get rid of the term with $\psi$ by showing the next lemma.

Lemma 11. Let $\psi$ be a $\mathcal{C}^{\infty}$ function with compact support. Then there exist admissible constants $C, C_{q}$ depending on $\psi$ such that, for $f$ a periodic integrable function supported in $(0,+\pi / 4)$ (modulo $2 \pi)$, the quantity

$$
A(x)=\frac{1}{L} \int_{-\pi L}^{\pi L}\left[b\left(x+\frac{l}{L}(s-x)\right) e^{i \frac{\mu}{L}(x-s)}-b(x)\right] f(s) \psi\left(\frac{x-s}{L}\right) d s
$$

is uniformly bounded, for $|x|<\pi$, by $C\|b\|_{L^{\infty}(\mathbb{T})}\|f\|_{L^{1}(\mathbb{T})}$. Moreover, it is bounded by $C_{q}\|b\|_{\Lambda_{\beta}(\mathbb{T})}$ when $f$ is a q-atom, with $\beta=1 / q-1$. 
Proof. $A(x)$ may be written as a scalar product $\frac{1}{L}\langle\tilde{f}, g\rangle$ on the real line, where $\tilde{f}$ is the function on the real line which coincides with $f$ on $(-L \pi,+L \pi)$ and is zero outside, while $g$ takes care of the other terms. Consider the first case, and remark that $\|\tilde{f}\|_{L^{1}(\mathbb{R})}=L\|f\|_{L^{1}(\mathbb{T})}$, while the norm of $g$ in $L^{\infty}(\mathbb{R})$ is bounded, up to a constant, by the norm of $b$ in $L^{\infty}(\mathbb{T})$. The conclusion follows at once. Assume now that $q<1$. Then $\tilde{f}$ is the sum of $L^{q}$-atoms of $H^{q}(\mathbb{R})$. On the other hand, one has $\|g\|_{\Lambda_{\beta}(\mathbb{R})} \leq C\|b\|_{\Lambda_{\beta}(\mathbb{T})}$, with $C$ admissible constant. We conclude, using the fact that the space $\Lambda_{\beta}(\mathbb{R})$ is the dual space of $H^{q}(\mathbb{R})$.

The main term of $T f(x)$ can be written as $\widetilde{T} f(x)+\bar{T} f(x)$, where

$$
\widetilde{T} f(x)=\int_{-\pi}^{\pi}\left[b\left(x+\frac{l}{L}(s-x)\right) e^{i \frac{\mu}{L}(x-s)}-b(x)\right] f(s) \frac{d s}{x-s}
$$

and

$$
\bar{T} f(x)=\int_{\pi<|s|<L \pi}\left[b\left(x+\frac{l}{L}(s-x)\right) e^{i \frac{\mu}{L}(x-s)}-b(x)\right] f(s) \frac{d s}{x-s} .
$$

Let us start with the first term.

Proposition 12. There exist admissible constants $C_{q}$ so that

$$
\|\widetilde{T}(f)\|_{L^{p}(-\pi,+\pi)} \leq C_{q}\|b\|_{\Lambda_{\alpha}(\mathbb{T})}
$$

when $q>1$ and $f$ is supported in the interval $\left(0,+\frac{\pi}{4}\right)$ and $\|f\|_{L^{q}(\mathbb{T})} \leq 1$, or when $q \leq 1$ and $f$ is a q-atom supported in $(0,+r)$, for some $r \leq \frac{\pi}{4}$.

Proof. We can deduce the required estimate from the analogue of Theorem 3 on the real line, which we now state. We define, for $\beta \in \mathbb{R}$, for $b \in \Lambda_{\alpha}(\mathbb{R})$ and $f \in H^{q}(\mathbb{R})$,

$$
\mathcal{H}_{\beta}(b, f)(x):=\int_{-\infty}^{\infty}[b(x+\beta(s-x))-b(x)] f(s) \frac{d s}{x-s} .
$$

We have the following proposition.

Proposition 13. Let $1<p<\infty, 0<q<p$ and $\alpha=\frac{1}{q}-\frac{1}{p}$. There exists a constant $C=C(\beta)>0$ so that, for any sufficiently smooth functions $b \in \Lambda_{\alpha}(\mathbb{R})$ and $f \in H^{q}(\mathbb{R})$

$$
\left\|\mathcal{H}_{\beta}(b, f)\right\|_{H^{p}(\mathbb{R})} \leq C\|f\|_{H^{q}(\mathbb{R})}\|b\|_{\Lambda_{\alpha}(\mathbb{R})} .
$$

Furthermore, $C(\beta)$ is uniformly bounded when $\beta$ lies in a compact set of $\mathbb{R}$.

Remark 14. Let us emphasize that this result may be seen as an elementary case of the Lacey-Thiele Theorem on the bilinear Hilbert transform (see [LT1, [LT2], LT3).

Proof. Let us again simplify the notation, and set $S(f):=\mathcal{H}_{\beta}(b, f)$. The kernel of $S$ is bounded, up to the constant $C|\beta|^{\alpha}\|b\|_{\Lambda_{\alpha}(\mathbb{R})}$, by the Riesz potential $|x-s|^{-1+\alpha}$, and the estimate follows directly from the classical estimates on Riesz potentials when $q>1$.

Let us now concentrate on $q \leq 1$, and assume that $f$ is an atom supported in the interval $I=(-r,+r)$ (we can reduce to this case, using invariance by translation as in Lemma 10. Using interpolation, it is sufficient to consider non-integer values of $\alpha$. We assume that $k<\alpha<k+1$. We write $S(f)=A_{1}+A_{2}$, with $A_{1}=S(f) \mathbb{1}_{(-2 r,+2 r)}$. 
We prove that both $A_{1}$ and $A_{2}$ are $L^{p}$-functions. To prove that $A_{1} \in L^{p}$, we remark that, because of the fact that $f$ has vanishing moments up to order $k$, we can replace the content of the bracket, in the definition of $S(f)$ as an integral given in (13), by

$$
b(x+\beta(s-x))-\sum_{j=0}^{k} \frac{b^{(j)}(x)}{j !} \beta^{j}(s-x)^{j} .
$$

This last quantity is bounded by $C|\beta|^{\alpha}|s-x|^{\alpha}\|b\|_{\alpha}$. We obtain that

$$
\left|A_{1}(x)\right| \leq C|\beta|^{\alpha} r^{\alpha-\alpha^{\prime}}\|b\|_{\alpha} \mathcal{I}_{\alpha^{\prime}}(|f|)(x),
$$

where $\mathcal{I}_{\alpha^{\prime}}$ denotes the fractional integral related to the Riesz potential $|x-s|^{-1+\alpha^{\prime}}$. We have chosen $\alpha^{\prime}<1$, such that $\frac{1}{p}=\frac{1}{\tilde{p}}-\alpha^{\prime}$ with $\tilde{p}>1$. Then

$$
\left\|A_{1}\right\|_{L^{p}} \leq C|\beta|^{\alpha} r^{\alpha-\alpha^{\prime}}\|b\|_{\alpha}\|f\|_{L^{\tilde{p}}}
$$

We conclude for $A_{1}$ using the fact that $f$ is a $q$-atom supported in $(-r,+r)$, that is, $\|f\|_{L^{\infty}(\mathbb{R})} \leq C r^{-1 / q}$.

To deal with $A_{2}$, we replace the content of the bracket of (13) differently. We write that $b(x+\beta(s-x))-b(x)$ is the sum of three terms, that is,

$$
\begin{gathered}
b((1-\beta) x+\beta s)-\sum_{j=0}^{k} \frac{b^{(j)}((1-\beta) x)}{j !} \beta^{j} s^{j} \\
+\left(\sum_{j=0}^{k} \frac{b^{(j)}((1-\beta) x)}{j !} \beta^{j} x^{j}-b(x)\right)+\sum_{j=1}^{k} \frac{b^{(j)}((1-\beta) x)}{j !} \beta^{j}\left(s^{j}-x^{j}\right) .
\end{gathered}
$$

The last term may be written as the product of $s-x$ with a polynomial in $s$ of degree less than $k$. Because of the condition on the moments of $f$, the corresponding term is zero. The first term is bounded by $r^{\alpha}|\beta|^{\alpha}\|b\|_{\Lambda_{\alpha}(\mathbb{R})}$, the second one by $|\beta|^{\alpha}\|b\|_{\Lambda_{\alpha}(\mathbb{R})}|x|^{\alpha}$. So, we can write $A_{2}$ as a sum of two terms, $A_{2}^{(1)}$ and $A_{2}^{(2)}$, corresponding to the two terms above. For $|x|>2 r,\left|A_{2}^{(1)}(x)\right|$ is bounded by $r^{\alpha}|\beta|^{\alpha}\|b\|_{\Lambda_{\alpha}(\mathbb{R})}\|f\|_{L^{1}(\mathbb{R})} /|x|$. The bound for its $L^{p}$ norm follows at once. For $A_{2}^{(2)}$, we use the well-known fact that the Hilbert transform of the $q$-atom $f$ is bounded by $C r^{k+2-1 / q} /|x|^{k+2}$ for $|x|>2 r$. We obtain the estimate

$$
\left|A_{2}^{(2)}(x)\right| \leq C|\beta|^{\alpha}\|b\|_{\Lambda_{\alpha}(\mathbb{R})} r^{k+2-1 / q}|x|^{\alpha-k-2}
$$

for $|x|>2 r$, from which we conclude at once.

This finishes the proof of Proposition 13 .

We conclude the proof of Proposition 12 using Proposition 13 for $\mathcal{H}_{\beta}(\tilde{b}, \tilde{f})$, with $\beta=\frac{l}{L}, \tilde{f}$ the function that is equal to $f$ on $(0, r)$ and vanishes elsewhere, and $\tilde{b}(x)=b(x) e^{-i \frac{\mu}{l} x}$ (it is elementary to show that $\tilde{b}$ belongs to $\Lambda_{\alpha}(\mathbb{R})$ with a Lipschitz norm bounded by $C\|b\|_{\Lambda_{\alpha}(\mathbb{T})}$. 
We now turn to the estimate of $\bar{T} f$. We prove the following.

Proposition 15. Let $p>1$. There exist admissible constants $C, C_{q}$ so that,

(1) if $f \in L^{1}(\mathbb{T})$ and is supported in the interval $\left(0,+\frac{\pi}{4}\right)$, then

$$
\|\bar{T}(f)\|_{L^{p}(-\pi,+\pi)} \leq C\|f\|_{L^{1}(\mathbb{T})}\|b\|_{L^{p}(\mathbb{T})} .
$$

(2) if $q<1$ and $f$ is a q-atom supported in $(0,+r)$, for some $r \leq \frac{\pi}{4}$, then

$$
\|\bar{T}(f)\|_{L^{p}(-\pi,+\pi)} \leq C_{q}\|b\|_{\Lambda_{\beta}(\mathbb{T})},
$$

with $\beta=1 / q-1$.

Proof. We may assume that $L \geq 2$, otherwise there is nothing to prove. We begin with the case $q \geq 1$. We will not use any compensation between the two terms involving $b$, so we replace $\bar{T}$ by $S$, where

$$
S(f)(x):=\int_{\pi<|t|<\pi L} b\left(\frac{k}{L} x+\frac{l}{L} t\right) e^{i \frac{\mu}{L}(x-t)} f(t) \frac{d t}{x-t} .
$$

We show (15) for $S(f)$ in place of $\bar{T}(f)$. The difference between both is of the same type as $S(f)$, except that $b$ is replaced by a constant. Now, we use the $2 \pi$-periodicity of $f$ to write $S(f)(x)$ as

$$
\int_{-\pi}^{\pi} f(t) \sum_{1 \leq|j|<L / 2} b\left(\frac{k}{L} x+\frac{l}{L}(t+2 \pi j)\right) e^{i \frac{\mu}{L}(x-t-2 \pi j)} \frac{d t}{x-2 \pi j-t} .
$$

We may replace $\frac{d t}{t+2 \pi j-x}$ by $\frac{d t}{2 \pi j}$ in the last integral. Indeed, the difference is bounded, up to a constant, by $1 / j^{2}$. Taking the sum, we get a quantity which is bounded, up to an admissible constant, by $\|b\|_{L^{p}(\mathbb{T})}\|f\|_{L^{1}(\mathbb{T})}$. Indeed, the constant comes from the fact that, for the periodic function $b$,

$$
\left\|b\left(\frac{k}{L} \cdot\right)\right\|_{L^{p}(-\pi,+\pi)} \leq C\left(1+\frac{L}{|k|}\right)^{\frac{1}{p}}\|b\|_{L^{p}(\mathbb{T})} .
$$

It remains to consider the term

$$
\int_{-\pi}^{\pi} f(t) \sum_{1 \leq|j|<L / 2} b\left(\frac{k}{L} x+\frac{l}{L}(t+2 \pi j)\right) \frac{e^{-i \frac{\mu}{L}(t+2 \pi j)}}{j} d t .
$$

Taking the $L^{p}$-norm on $(-\pi,+\pi)$ and using Minkowski inequality, it is sufficient to show that $\left\|B\left(\frac{k}{L} \cdot, t\right)\right\|_{L^{p}(-\pi,+\pi)} \leq C\|b\|_{L^{p}(\mathbb{T})}$, with $C$ an admissible constant which is independent of $t$, where

$$
B(x, t):=\sum_{1 \leq|j|<L / 2} b\left(x+\frac{l}{L}(t+2 \pi j)\right) \frac{e^{-2 \pi i \frac{\mu j}{L}}}{j} .
$$

We use the inequality (17) written for the function $B(\cdot, t)$ to reduce to an inequality for this last function. We will use transference to conclude. Let $\Theta_{j}$ be the strongly continuous representation of $\mathbb{Z}$ in $L^{p}(\mathbb{T})$ given by

$$
\Theta_{j} b(t)=b\left(t+\frac{l}{L} 2 \pi j\right)
$$


It is clear that $B(\cdot, t)$ is obtained from $b\left(\cdot+\frac{l t}{L}\right)$ when using the operator

$$
\sum_{1 \leq|j|<L / 2} \frac{e^{-2 \pi i \frac{\mu j}{L}}}{j} \Theta_{j}
$$

The theory of tranference (see $\mathrm{CW}$ ) allows us to conclude that it is a uniformly bounded operator on $L^{p}(\mathbb{T})$ once we know that the operator given by the convolution by the sequence $c_{j}:=e^{-2 \pi i \frac{\mu j}{L}} / j$ for $1 \leq|j|<L / 2, c_{j}=0$ otherwise, is bounded on $\ell^{p}(\mathbb{Z})$. This, again, is classical: the convolution operator on $\ell^{p}(\mathbb{Z})$ associated to the sequence $(1 / j)_{|j| \geq 1}$ is a bounded operator on $\ell^{p}(\mathbb{Z}), p>1$, as the discrete analogue of the Hilbert transform. The same also holds for the truncated sequence $\left(c_{j}^{L}\right)$ where $c_{j}^{L}:=1 / j$ for $1 \leq|j|<L / 2$, and $c_{j}^{L}=0$ otherwise, with an operator norm bounded independently of $L$. The same is also valid for the sequence $c_{j}$ given above: we may write this last operator in terms of the conjugate of the previous one under the action of the multiplication by $e^{-2 \pi i \frac{\mu j}{L}}$. Finally,

$$
\|B(\cdot, t)\|_{L^{p}(\mathbb{T})} \leq C\left\|b\left(\cdot+\frac{l t}{L}\right)\right\|_{L^{p}(\mathbb{T})}=C\|b\|_{L^{p}(\mathbb{T})} .
$$

This finishes the proof of (15).

Let us now prove (16). For simplicity, we only consider the case when $\beta$ is not an integer, which is sufficient for our purpose. We assume that $f$ is an atom supported in $(0, r)$. We write $\bar{T}(f)(x)$ as

$$
\int_{-\pi}^{\pi} f(t) \sum_{1 \leq|j|<L / 2}\left[b\left(\frac{k}{L} x+\frac{l}{L}(t+2 \pi j)\right) e^{i \frac{\mu}{L}(x-t-2 \pi j)}-b(x)\right] \frac{d t}{x-2 \pi j-t} .
$$

As in the proof of Proposition [13, we write the content of the bracket as a sum of terms. Let us first define

$$
\tilde{b}(x):=b(x) e^{-i \frac{\mu}{l} x} .
$$

As we already pointed out, it is elementary to see that $\tilde{b}$ is in $\Lambda_{\beta}(\mathbb{R})$ with a norm bounded by $C\|b\|_{\Lambda_{\beta}(\mathbb{T})}$. Then, the content of the bracket is equal to $e^{i \frac{\mu}{l} x} \tilde{B}_{j}(x, t)$ with $\tilde{B}_{j}(x, t)=\tilde{B}_{j}^{(1)}(x, t)+\tilde{B}_{j}^{(2)}(x, t)+\tilde{B}_{j}^{(3)}(x, t)$, with

$$
\begin{aligned}
& \tilde{B}_{j}^{(1)}(x, t)=\tilde{b}\left(\frac{k}{L} x+\frac{l}{L}(t+2 \pi j)\right)-\sum_{s=0}^{[\beta]} \tilde{b}^{(s)}\left(\frac{k}{L} x+\frac{l}{L} 2 \pi j\right)\left(\frac{l}{L}\right)^{s} \frac{t^{s}}{s !}, \\
& \tilde{B}_{j}^{(2)}(x, t)=\sum_{s=0}^{[\beta]} \tilde{b}^{(s)}\left(\frac{k}{L} x+\frac{l}{L} 2 \pi j\right)\left(\frac{l}{L}\right)^{s} \frac{(x-2 \pi j)^{s}}{s !}-\tilde{b}(x), \\
& \tilde{B}_{j}^{(3)}(x, t)=\sum_{s=0}^{[\beta]} \tilde{b}^{(s)}\left(\frac{k}{L} x+\frac{l}{L} 2 \pi j\right)\left(\frac{l}{L}\right)^{s} \frac{\left(t^{s}-(x-2 \pi j)^{s}\right)}{s !} .
\end{aligned}
$$

The last term may be written as the product of $x-2 \pi j-t$ with a polynomial of degree less than $k$ in $t$. Because of the condition on the moments of $f$, the corresponding term is zero.

Let us then consider the second term, which is bounded by $C|x-2 \pi j|^{\beta}\|b\|_{\Lambda_{\beta}(\mathbb{T})}$, where $C$ is an admissible constant. To get a bound for the corresponding term in $\bar{T}(f)(x)$, we use the fact that the Hilbert transform of $f$, at the point $x-2 \pi j$, can be 
bounded by $C r^{[\beta]+1-\beta} /|j|^{[\beta]+2}$. We conclude, using the fact that $\sum_{|j| \geq 1}|j|^{\beta-[\beta]-2}$ is finite, and $r^{[\beta]+1-\beta}$ is bounded.

Let us finally consider the first term, which is bounded by $C r^{\beta}\|b\|_{\Lambda_{\beta}(\mathbb{T})}$, with $C$ an admissible constant. We proceed exactly as in the proof of (15), using the fact that the $L^{1}$-norm of $f$ is bounded by $r^{-\beta}$. We may as well replace $t+2 \pi j-x$ by $2 \pi j$ in the last integral in the denominator. Moreover, one easily verifies that $e^{i \frac{\mu}{l} x} \tilde{B}_{j}^{(1)}(x, t)$ can be written as $e^{i \frac{\mu}{L} x} e^{-2 \pi i \frac{\mu j}{L}} c\left(\frac{k}{L}\left(x+2 \pi \frac{l}{k} j\right), t\right)$, where the function $c$ is periodic in the first variable $x$, and is bounded by $C r^{\beta}\|b\|_{\Lambda_{\beta}(\mathbb{T})}$, with $C$ an admissible constant which is independent of $t$. After this point, the proof is exactly the same as the one of (15), considering the sum

$$
\sum_{1 \leq|j|<L / 2} c\left(\frac{k}{L}\left(x+2 \pi \frac{l}{k} j\right), t\right) \frac{e^{-2 \pi i \frac{\mu j}{L}}}{j} .
$$

We will not repeat the proof here, and leave the details for the reader. This finishes the proof of formula (11), as well as the proof of Theorem 1 .

\section{REFERENCES}

[BB] Bonami, A. and Bruna, J. On truncations of Hankel and Toeplitz operators. Publ. Mat. 43 no. 1, 235-250 (1999). MR1697523 (2000c:47046)

[BL] Bergh, J. and Löfström, J. Interpolation spaces. An introduction. Grundlehren der Mathematischen Wissenschaften, No. 223. Springer-Verlag, Berlin-New York, 207 pp (1976). MR0482275 (58:2349)

[CM] Coifman, R.R. and Meyer, Y. On commutators of singular integrals and bilinear singular integrals, Trans. Am. Math. Soc., 212, 315-331 (1975). MR0380244 (52:1144)

[CW] Coifman, R. R. and Weiss, G. Transference methods in analysis. Conference Board of the Mathematical Sciences Regional Conference Series in Mathematics, No. 31. American Mathematical Society, Providence, R.I., (1976). MR0481928 (58:2019)

[D] Duren, P. L. Theory of $H^{p}$ spaces. Pure and Applied Mathematics, Vol. 38 Academic Press, New York-London 258 pp (1970). MR0268655 (42:3552)

[GR] García-Cuerva, J. and Rubio de Francia, J. L. Weighted norm inequalities and related topics. North-Holland Mathematics Studies, 116. Notas de Matemática [Mathematical Notes], 104. North-Holland Publishing Co., Amsterdam, 604 pp (1985). MR0807149 (87d:42023)

[GB] Gasch, J.E. and Gilbert, J. E. Triangularization of Hankel operators and the bilinear Hilbert transform, Contemp. Math., 247, 235-248, (1999). MR.1738092 (2001b:47044)

[GraK] Grafakos, L. and Kalton, N. Multilinear Calderón-Zygmund operators on Hardy spaces, Collect. Math., 52 (2), 169-179 (2001). MR.1852036 (2002k:42032)

[GraL] Grafakos, L. and Li, X. Uniform bounds for the bilinear Hilbert transforms. I. Ann. of Math. (2) 159 (2004), no. 3, 889-933. MR2113017 (2006e:42011)

[GraT] Grafakos, L. and Torres, R.H. Multilinear Calderón-Zygmund theory, Adv. in Math., 165, 124-164 (2002). MR 1880324 (2002j:42029)

[GreK] Grellier, S. and Kacim, M. Truncation of multilinear Hankel operators, preprint (2003).

[KS] Kenig, C.E. and Stein, E.M. Multilinear estimates and fractional integration, Math. Research Lett., 6, 1-15 (1999). MR1682725 (2000k:42023a)

[L] Lacey, M. The bilinear maximal function maps in $L^{p}$ for $2 / 3<p \leq 1$, Ann. of Math., 151(2), 35-57 (2000). MR:1745019 (2001b:42015)

[LT1] Lacey, M. and Thiele, C. $L^{p}$ estimates on the bilinear Hilbert transform, $2<p<\infty$, Ann. of Math., 146, 683-724 (1997). MR1491450 (99b:42014)

[LT2] Lacey, M. and Thiele, C. M. On Calderón's conjecture for the bilinear Hilbert transform. Proc. Natl. Acad. Sci. USA 95 , no. 9, 4828-4830 (1998) MR.1619285 (99e:42013)

[LT3] Lacey, M. and Thiele, C. On Calderón's conjecture, Annals of Math., 149, 475-496 (1999). MR1689336 (2000d:42003) 
[MTT] Muscalu, C., Tao, T. and Thiele, C. Multilinear operators given by singular multipliers, J. Am. Math. Soc., 15, 469-496 (2002). MR.1887641 (2003b:42017)

[S] Stein, E.M. Harmonic Analysis: Real Variable Methods, Orthogonality, and Oscillatory Integrals, Princeton University Press, Princeton, NJ (1993). MR.1232192 (95c:42002)

mapmo, Université d'Orléans, Faculté des Sciences, Département de Mathématiques, BP 6759, F 45067 Orleans CÉdex 2, France

E-mail address: bonami@labomath.univ-orleans.fr

Mapmo, Université d'Orléans, Faculté des Sciences, Département de MathémaTiques, BP 6759, F 45067 Orleans CÉdex 2, France

E-mail address: grellier@labomath.univ-orleans.fr

Université Saint-Joseph, Rue de Damas, Beirut 1104-2020, Lebanon

E-mail address: kacim76@hotmail.com 